\section{Analysis of long- term outcomes for combined pars plana vitrectomy (PPV) and glaucoma tube shunt surgery in eyes with advanced glaucoma}

A Gandhi', DM Miller², JM Zink², AK Khatana², CD Riemann², MR Petersen², RE Foster ${ }^{2}$ and RA Sisk ${ }^{2}$
${ }^{1}$ Department of Ophthalmology, University of Cincinnati College of Medicine, Cincinnati, $\mathrm{OH}$, USA

${ }^{2}$ Cincinnati Eye Institute, Cincinnati, $\mathrm{OH}$, USA

Correspondence: DM Miller, Cincinnati Eye Institute, 1945 CEl Drive, Cincinnati, OH 45242, USA.

Tel: +1 513984 5133;

Fax: +1 513984 9550;

E-mail: dmiller@

cincinnatieye.com

Received: 16 May 2013 Accepted in revised form: 25 October 2013;

Published online:

13 December 2013

\begin{abstract}
Purpose To analyze 12- and 24-month visual acuity, intraocular pressure, and complications associated with combined pars plana vitrectomy (PPV) and glaucoma tube shunt placement in eyes with glaucoma. Patients and methods A retrospective chart review was performed of patients with advanced glaucoma who underwent combined PPV and tube shunt surgery from 2006 to 2010. A minimum of 12 months of follow-up was required for their inclusion in the study. Visual acuity, intraocular pressure, complications, and number of glaucoma medications at $\mathbf{1}$ and 2 years postoperatively were analyzed.

Results Twenty-eight eyes met the inclusion and exclusion criteria. Baseline visual acuity was $20 / 200$ or worse in $14 / 28$ eyes $(50.0 \%$ ) and $20 / 40$ or better in $2 / 28$ eyes $(\mathbf{7 . 1 \%})$. Visual acuity remained $20 / 200$ or worse in $50.0 \%$ $(P=0.921)$ and $44.4 \%(P=0.973)$ of eyes after 1 and 2 years postoperatively, respectively. At baseline, the mean intraocular pressure was $30.4 \mathrm{~mm} \mathrm{Hg}$. There was significant improvement in mean IOP at 1 year $(14.7 \mathrm{~mm} \mathrm{Hg}, P=0.001)$ and at 2 years (15.2 $\mathrm{mm} \mathrm{Hg}, P=0.001$ ) postoperatively. Baseline number of glaucoma medications averaged $3.0 \pm 1.09$ (SD), and improved to $1.8 \pm 1.28(\mathrm{SD})$ at 1 year $(P=0.0002)$ and to $1.4 \pm 1.33$ at 2 years $(P<0.0001)$

postoperatively.

Conclusion In this retrospective interventional case series, surgical management of advanced glaucoma with a combination of PPV and glaucoma tube
\end{abstract}

shunt resulted in significantly reduced IOP and glaucoma medications at 1 and 2 years postoperatively.

Eye (2014) 28, 290-295; doi:10.1038/eye.2013.263; published online 13 December 2013

Keywords: pars plana vitrectomy; glaucoma tube shunt; glaucoma; intraocular pressure

\section{Introduction}

Glaucoma tube shunts serve as a non-healing permanent fistula by which aqueous fluid drains from the anterior chamber to the subconjunctival space. ${ }^{1,2}$ Tube shunts are particularly beneficial in eyes that have had prior unsuccessful laser or filtration surgeries, or when traditional filtration surgery is contraindicated due to ocular anatomy and pathology. ${ }^{3}$ Conventional filtration surgery has reduced success in secondary glaucoma, aphakic glaucoma, neovascular glaucoma, and developmental glaucoma. ${ }^{2}$ Tube shunts are indicated in eyes with excessive conjunctival scarring that would reduce the success of a repeated trabeculectomy, ${ }^{4-6}$ in eyes with irregularities in the iridocorneal angle, 5,6 in inflammatory glaucoma, ${ }^{2}$ and in the presence of a keratoprosthesis. 1,7

In selective cases, the insertion of the drainage tube into the anterior chamber is contraindicated because of a shallow anterior chamber, ${ }^{8-10}$ vitreous prolapse, pseudophakia/ aphakia, ${ }^{11}$ complicated glaucoma with corneal disease and angle neovascularization, ${ }^{12-14}$ anterior chamber pathology such as peripheral 
synechiae secondary to inflammation, corneal disease, previous corneal surgeries (penetrating keratoplasty or kerato prosthesis), ${ }^{15,16}$ and failed prior

trabeculectomies. ${ }^{3,7,17-19}$ In such cases, the glaucoma tube shunt can be inserted through the pars plana with simultaneous pars plana vitrectomy (PPV). A PPV may also be indicated to address vitreous prolapse into the anterior chamber when the tube is positioned in the anterior chamber. In addition, PPV may be required for posterior segment tube positioning or to address posterior segment pathology such as retinal detachment, retained lens fragments, proliferative diabetic retinopathy, media opacities, neovascularization, or epiretinal membrane. ${ }^{12}$

The purpose of this study was to report long-term visual acuity outcomes, intraocular pressure outcomes, and complications associated with combined PPV and glaucoma tube shunt placement in eyes with glaucoma.

\section{Patients and methods}

A retrospective review of combined glaucoma and PPV surgeries was approved by the University of Cincinnati IRB. A retrospective chart review was performed of patients with advanced glaucoma who underwent combined PPV and tube shunt surgery between 2006 and 2010. Surgeries were performed by one of two glaucoma surgeons (AKK and JMZ) and one of five vitreoretinal surgeons (REF, DMM, MRP, CDR, and RAS) at the Cincinnati Eye Institute. A minimum of 12 months of follow-up was required for inclusion in the study. Staged surgeries were excluded. A total of 28 eyes of 28 patients met the inclusion and exclusion criteria.

Patient medical records were reviewed and data were recorded for patient demographics (age, gender, eye), ocular surgical history, indication for tube shunt placement, indications for PPV, preoperative Snellen visual acuity (VA), preoperative intraocular pressure (IOP), and preoperative glaucoma medications. The preoperative visual acuity, IOP, and number of glaucoma medications used were the last recorded values prior to surgery. Primary outcome measures were Snellen visual acuity, IOP, number of glaucoma medications, and postoperative complications at 1 and 2 years postoperatively. The types of glaucoma drainage implants used included the Baerveldt ( 250 and $350 \mathrm{~mm}^{2}$ ) (Advanced Medical Optics, Santa Ana, CA, USA), Ahmed-S2 (New World Medical, Rancho Cucamonga, CA, USA), and Molteno-3 (Molteno Ophthalmic Limited, Dunedin, New Zealand). Their position in the anterior chamber or vitreous cavity was recorded. The instrumentation $(20,25$, or 23) used for PPV was noted.

All patients underwent either general anesthesia or monitored anesthesia care with retrobulbar or subconjunctival anesthesia. Periocular skin was prepped with $5 \%$ povidone-iodine, followed by a drop in the inferior fornix. After the eye was draped in standard manner and an eyelid speculum was placed, a fornixbased conjunctival flap was fashioned in the quadrant in which the drainage implant was to be inserted. The tube was ligated externally with a 7-0 Vicryl anterior to the plate to completely occlude the tube. Complete occlusion was confirmed by trying to flush BSS through the tube with a 30-gauge cannula. The plate of the tube shunt was sutured to the sclera just posterior to the muscle insertions with a 9-0 nylon simple interrupted suture through the anterior anchoring holes in the plate. The free end of the tube was then tucked underneath the plate so that it does not interfere with the vitrectomy.

Every PPV surgery was performed using the Accurus Vitreoretinal Surgical System or Constellation Vitreoretinal Surgical System (Alcon Laboratories, Inc., Fort Worth, TX, USA) and either Xenon (Alcon Laboratories, Inc.) or Photon (Synergetics, O'Fallon, MO, USA) light sources. Either a 25-gauge or a 23-gauge trocar/cannula system was used to place cannulas in a beveled manner either through displaced conjunctiva or directly through the sclera if within the area of the peritomy. Cannulas were placed inferotemporally, superotemporally, and superonasally $3 \mathrm{~mm}$ posterior to the limbus for pseudophakic eyes and $4 \mathrm{~mm}$ for phakic eyes. In some cases, a standard 3-port 20-gauge vitrectomy was utilized. Sclerotomies were placed as in cannula-based surgery. Wide-angle fundus visualization was achieved using either the BIOM noncontact widefield imaging system (Oculus, Munich, Germany) or the AVI contact panoramic viewing system (Advanced Visual Systems, Inc., New York, NY, USA). The surgical procedure varied depending on the indication for the surgery, the severity of the pathology, and the individual preference of the surgeon. A core vitrectomy was performed. The posterior hyaloid membrane and posterior cortical vitreous were dissected from the macula using aspiration and/or manual membrane peeling. Peripheral vitrectomy was then performed. Aggressive peripheral vitreous dissection was performed in eyes with pars plana tube placement with particular care taken to perform a complete vitrectomy in the quadrants adjacent to the pars plana tube placement. Some patients had a full or partial air-fluid exchange with a silicone-tipped extrusion cannula. At the conclusion of the vitrectomy, the cannulas were removed and the superior sclerotomies were sutured. The infusion line was left in place for the remainder of the glaucoma surgery.

After the pars plana vitrectomy was performed, the tube was trimmed and a 23-gauge needle was used to create a new sclerotomy track through the pars plana. A similar procedure was used for placing the tube in the anterior chamber. The tube was inserted into the vitreous 
cavity or anterior chamber and fixed to the sclera with 9-0 nylon or 8-0 vicryl sutures. Either a corneal or scleral patch graft was trimmed and sutured to the sclera to cover the tube with 8-0 vicryl sutures. Fenestrations were made in the tube as needed to allow pressure lowering depending on surgeon preferences. The conjunctiva was closed with either an 8-0 or a 9-0 vicryl suture. The infusion cannula was then removed and the sclerotomy sutured with 7-0 vicryl.

A two-tailed paired Student $t$-test was performed to evaluate whether a statistically significant change $(P<0.05)$ had occurred between preoperative and postoperative IOP and visual acuity. The Snellen visual acuity values at baseline and at 1 and 2 years postoperatively were converted to LogMAR prior to performing the $t$-test. The Fisher Exact test was performed to evaluate whether a statistically significant change occurred between preoperative and postoperative number of glaucoma medications.

\section{Results}

Twenty-eight eyes of 28 patients met the inclusion and exclusion criteria. The mean patient age was 57 years. Baseline patient demographics are summarized in Table 1. All 28 eyes had 1 year of follow-up, and 18/28 eyes had 2 years of follow-up.

Indications for tube shunt placement were uncontrolled glaucoma due to chronic angle closure glaucoma (11/28, $39.3 \%)$, primary open angle glaucoma $(6 / 28,21.4 \%)$, neovascular glaucoma $(6 / 28,21.4 \%)$, steroid-induced glaucoma $(3 / 28,10.7 \%)$, chemical injury-induced glaucoma $(1 / 28,3.6 \%)$, and aphakic glaucoma $(1 / 28,3.6 \%)$.

Most of these eyes had undergone prior intraocular surgeries, which are summarized in Table 1 . Previous surgeries included cataract surgery $(8 / 28,28.6 \%)$, penetrating keratoplasty $(7 / 28,25.0 \%)$, Boston keratoprosthesis $(3 / 28,10.7 \%)$, and vitrectomy $(9 / 28$, $32.1 \%)$. Of the 28 eyes, 1 eye $(3.6 \%)$ had undergone failed surgeries previously for lowering intraocular pressure (trabeculectomy or tube placement).

The indications for PPV were planned posterior segment tube placement $(13 / 28,46.4 \%)$ or anterior chamber tube placement with vitreous prolapse $(10 / 28$, $35.7 \%)$, epiretinal membrane $(3 / 28,10.7 \%)$, or tractional retinal detachment $(3 / 43,6.9 \%)$. These indications are summarized in Table 1.

All eyes underwent combined PPV and tube shunt surgery. Out of the 28 patients, $21(75.0 \%)$ received the Baerveldt tube shunt. Molteno-3 and Ahmed-S2 shunts were utilized in the remainder. The glaucoma tubes were positioned in the posterior segment in $13 / 28$ eyes (46.4\%) and in the anterior chamber in 15/28 (53.6\%) eyes. More than half of the eyes underwent cannula-based
PPV: 25-gauge (17/28, 60.7\%), 20-gauge $(8 / 28,28.6 \%)$, and 23-gauge PPV $(3 / 28,10.7 \%)$.

Baseline and postoperative visual acuity values are summarized in Table 2 . At baseline, visual acuity was $20 / 200$ or worse in $14 / 28(50.0 \%$ ) eyes and $20 / 80$ to $20 / 200$ in $4 / 28(14.3 \%)$ eyes. Only two eyes $(7.1 \%)$ had a visual acuity of $20 / 40$ or better. There was no statistical difference between baseline visual acuity (20/200 or worse, $50.0 \%$ ) and visual acuity at 1 year (20/200 or worse, $50.0 \%$, $P=0.921)$ and 2 years $(20 / 200$ or worse, $44.4 \%, P=0.973)$.

Table 1 Patient demographics

\begin{tabular}{lc}
\hline Age (years) & 57 \\
Mean & $14-86$ \\
Range & \\
& \\
Gender & Number of patients \\
M & $10(35.7 \%)$ \\
F & $18(64.3 \%)$ \\
& \\
Eye & \\
R & $15(53.6 \%)$ \\
L & $13(46.4 \%)$ \\
& \\
Primary diagnosis & \\
Chronic angle closure glaucoma & $11(39.3 \%)$ \\
Neovascular glaucoma & $6(21.4 \%)$ \\
Primary open angle glaucoma & $6(21.4 \%)$ \\
Steroid-induced glaucoma & $3(10.7 \%)$ \\
Aphakic glaucoma & $1(3.6 \%)$ \\
Chemical injury-induced glaucoma & $1(3.6 \%)$ \\
Prior surgeries & \\
Corneal (PK, K-pro, EK) & \\
Vitrectomy & $12(42.9 \%)$ \\
Cataract & $9(32.1 \%)$ \\
Glaucoma (trabeculectomy, tube) & $10(35.7 \%)$ \\
Indications for PPV & $1(3.6 \%)$ \\
Posterior segment tube & \\
Vitreal prolapse into AC & \\
Epiretinal membrane & $13(46.4 \%)$ \\
Tractional retinal detachment & $10(35.7 \%)$ \\
\hline Abbreviations: EK, endothelial keratoplasty; K-pro, Boston keratoprosthesis; \\
PK, penetrating keratoplasty. & $3(10.7 \%)$ \\
& $2(7.1 \%)$ \\
\hline
\end{tabular}

Table 2 Visual acuity outcomes

\begin{tabular}{lccc}
\hline Snellen visual acuity & $\begin{array}{c}\text { Baseline } \\
(\mathrm{n}=28)\end{array}$ & $\begin{array}{c}\text { After 1 year } \\
(\mathrm{n}=28)\end{array}$ & $\begin{array}{c}\text { After 2 years } \\
(\mathrm{n}=18)\end{array}$ \\
\hline $20 / 40$ or better & $2(7.1 \%)$ & $2(7.1 \%)$ & $1(5.6 \%)$ \\
$<20 / 40$ to $20 / 80$ & $8(28.6 \%)$ & $6(21.4 \%)$ & $6(33.3 \%)$ \\
$<20 / 80$ to $20 / 200$ & $4(14.3 \%)$ & $6(21.4 \%)$ & $3(16.7 \%)$ \\
$\begin{array}{l}\text { Worse than } 20 / 200 \\
\text { Mean LogMAR }\end{array}$ & $14(50.0 \%)$ & $14(50.0 \%)$ & $8(44.4 \%)$ \\
$\begin{array}{l}\text { visual acuity } \\
\text { Range LogMAR }\end{array}$ & 0.1 .32 & $1.30(P=0.921)$ & $1.33(P=0.973)$ \\
\hline
\end{tabular}


Baseline and postoperative intraocular pressure values are summarized in Table 3. The majority of eyes had moderate to advanced optic nerve cupping at baseline. Patients who received a prior Boston Keratoprosthesis had intraocular pressure values estimated by palpation, and therefore the pressure estimates of three eyes were not included in the intraocular pressure analysis. The mean preoperative intraocular pressure was $30.4 \pm 9.8$ (SD) $\mathrm{mm} \mathrm{Hg}$. Preoperative intraocular pressures were elevated in 6/25 (24.0\%) cases with an IOP between 21 and $30 \mathrm{~mm} \mathrm{Hg}$ and in 12/25 (48.0\%) eyes with an IOP between 31 and $40 \mathrm{~mm} \mathrm{Hg}$. Postoperatively, intraocular pressures reduced significantly to a mean value of $14.7 \pm 6.0$ (SD) $\mathrm{mm} \mathrm{Hg}$ at 1 year $(P<0.0001)$ and $15.2 \pm 10.3(\mathrm{SD}) \mathrm{mm} \mathrm{Hg}$ at 2 years postoperatively $(P<0.0001)$. After 1 year, $15 / 25$ eyes $(60.0 \%)$ had an intraocular pressure between 11 and $20 \mathrm{~mm} \mathrm{Hg}$, and only 5/25 eyes (20.0\%) had an intraocular pressure between 21 and $30 \mathrm{~mm} \mathrm{Hg}$. Similar results for intraocular pressure were seen at 2 years postoperatively. No differences were seen in intraocular pressure values at baseline, 1 year, or 2 years between eyes with anteriorly or posteriorly placed tube shunts (data not shown).

Preoperatively, most patients were on maximally tolerated topical and/or systemic medications, with 22/28 (78.6\%) eyes receiving three or more glaucoma medications (Table 4 ). The mean number of medications was $3.0 \pm 1.09$ (SD) preoperatively. The mean number of glaucoma medications was reduced to $1.8 \pm 1.28$ (SD) at 1 year postoperatively $(P<0.0001)$ and to $1.4 \pm 1.33(\mathrm{SD})$ at 2 years postoperatively $(P<0.0001)$. The number of glaucoma medications is summarized in Table 4. Only 10/28 (35.7\%) eyes at 1 year required 3 or more glaucoma medications and 18/28 (64.3\%) eyes required 2 or fewer glaucoma medications. Similar findings were seen at 2 years postoperatively.

Postoperative complications were observed in $4 / 28$ (14.3\%) cases. Complications included tube erosions (2 eyes), hypotony (1 eye), and retinal detachment (1 eye). The patient with chronic hypotony postoperatively had a history of aniridia and prior keratoprosthesis. Her surgery consisted of a 20-gauge vitrectomy combined with posterior segment tube shunt placement. The two patients with tube erosions had posterior segment tube shunt

Table 3 Intraocular pressure outcomes

\begin{tabular}{lccc}
\hline $\begin{array}{l}\text { Intraocular } \\
\text { pressure }(\mathrm{mm} \mathrm{Hg})\end{array}$ & $\begin{array}{c}\text { Baseline } \\
(\mathrm{n}=25)\end{array}$ & $\begin{array}{c}\text { After 1 year } \\
(\mathrm{n}=25)\end{array}$ & $\begin{array}{c}\text { After 2 years } \\
(\mathrm{n}=17)\end{array}$ \\
\hline $0-10$ & $0(0 \%)$ & $8(32.0 \%)$ & $5(27.8 \%)$ \\
$11-20$ & $5(20.0 \%)$ & $15(60.0 \%)$ & $10(55.6 \%)$ \\
$21-30$ & $6(24.0 \%)$ & $5(20.0 \%)$ & $2(11.1 \%)$ \\
$31-40$ & $12(48.0 \%)$ & $0(0 \%)$ & $0(0 \%)$ \\
$41-50$ & $2(8.0 \%)$ & $0(0 \%)$ & $1(5.6 \%)$ \\
Mean IOP & 30.4 & $14.7(P<0.0001)$ & $15.2(P<0.0001)$ \\
Range IOP & $13-54$ & $2-27$ & $2-48$ \\
\hline
\end{tabular}

placement in eyes with prior keratoprosthesis surgery for severe ocular surface disease. Retinal detachment developed in one patient who had undergone 25-gauge vitrectomy and anterior chamber tube shunt placement. The retinal detachment was successfully repaired with vitrectomy,endolaser, and gas tamponade.

\section{Discussion}

The management of eyes with glaucoma in the setting of scarred conjunctiva, ${ }^{4-6}$ prior failed filtration surgeries, ${ }^{3,17,18}$ corneal pathology, ${ }^{19}$ inflammation, ${ }^{20}$ or neovascularization ${ }^{13,14}$ is complex. The glaucoma tube shunt has been shown to be beneficial in such cases. ${ }^{21}$ Pars plana tube insertion can be particularly beneficial in eyes with a shallow anterior chamber, corneal disease, previous penetrating keratoplasty, or keratoprosthesis. ${ }^{12}$ The details of a patient's clinical presentation determine whether anterior or posterior positioning is optimal. The unique interplay of the possible coexisting ocular pathologies and past ocular surgical history requires surgical techniques to be individualized for each patient.

This current series reports the long-term results on eyes that have undergone combined PPV and glaucoma drainage tube placement in the anterior or posterior segment. The baseline and postoperative visual acuity outcomes at 1 and 2 years were not statistically different. Scott and associates reported that in 31 patients $(77.5 \%)$ Snellen visual acuity was stable or improved 1 year postoperatively after a combined PPV and glaucoma drainage implant for refractory glaucoma. This limitation to visual acuity has been attributed to prior underlying ocular disease ${ }^{12}$ and was a limiting factor in this study as well. The mean preoperative intraocular pressure was $29.5 \mathrm{~mm} \mathrm{Hg}$ with a mean number of 3 glaucoma medications. One year postoperatively, the mean IOP reduced to $15.2 \mathrm{~mm} \mathrm{Hg}$ with a mean of 1.5 medications. Two years postoperatively, the mean IOP remained stable at $13.6 \mathrm{~mm} \mathrm{Hg}$ and the number of glaucoma medications reduced to 1.3. A dramatic decrease was seen in the

Table 4 Glaucoma medications

\begin{tabular}{lcr}
\hline Number of medications & Number of patients & \\
\hline $\begin{array}{l}\text { Preoperative } \\
\text { Two or less }\end{array}$ & $6(21.4 \%)$ & \\
Three or more & $22(78.6 \%)$ & \\
& & \\
Number of medications & After 1 year & After 2 years \\
& $(\mathrm{P}=0.0002)$ & $(\mathrm{P}<0.0001)$ \\
\hline $\begin{array}{l}\text { Postoperative } \\
\text { Two or less } \\
\text { Three or more }\end{array}$ & $18(64.3 \%)$ & $13(72.2 \%)$ \\
\hline
\end{tabular}


number of eyes with an IOP between 21 and $30 \mathrm{~mm} \mathrm{Hg}$ : from $30.3 \%$ of patients preoperatively to $18.2 \%$ of patients 1 year postoperatively. The downward shift in IOP along with the decrease in the number of glaucoma medications postoperatively indicates the success of this procedure in reducing intraocular pressure in the long term.

The complications encountered in this series were tube erosions in 3 eyes, hypotony in 1 eye early postoperatively, and retinal detachment in 1 eye. Postoperative hypotony often occurs if the sclerotomy is not tightly closed. The leakage has been observed to occur more often when the tube passes through a needle tract of 21-gauge or larger. ${ }^{22}$ One of the patients in the series with hypotony had a 20-gauge PPV and the other had a 25-gauge PPV. The possible complications of a glaucoma drainage implant depend upon the location of insertion. Studies have shown an increased prevalence of displacement of the drainage tube, iritis, conjunctival erosion with tube exposure, prolapsed vitreous, corneal endothelium functional deterioration, and worsening of cataracts in tube shunts placed through the anterior chamber. $3,13,22$ In contrast, an increase in retinal detachment, tube obstruction by vitreous, and hypotony are seen in tube shunts placed through the posterior segment. ${ }^{2,13,22}$

Limitations of this current series include its retrospective design, lack of controls, and smaller sample size with 43 eyes. In this study, we showed that combined PPV and glaucoma drainage tube implantation can effectively reduce IOP and the number of required glaucoma medications in eyes refractory to medical and/ or prior surgical therapy with concurrent anterior or posterior segment pathologies.

\section{Summary}

What was known before

- A relatively small number of papers have been published on combined vitrectomy and glaucoma drainage implant surgery.

- Eyes undergoing this procedure typically have multiple ophthalmic diseases and extremely guarded prognosis.

- Most prior published series on this topic have a relatively short follow-up.

\section{What this study adds}

- This study adds long-term follow-up data for eyes undergoing combined vitrectomy and glaucoma drainage implantation.

- It is the first series to demonstrate the long-term outcomes of smaller-gauge vitrectomy ( -25 and -23-gauge) instrumentation in managing these cases.

- This study enhances the data on this topic in the published literature, as it is relatively rare.

\section{Conflict of interest}

The authors declare no conflict of interest.

\section{References}

1 Kirkness CM, Ling Y, Rice NS. The use of silicone drainage tubing to control post-keratoplasty glaucoma. Eye (Lond) 1988; 2(Pt 5): 583-590.

2 Sherwood MB, Joseph NH, Hitchings RA. Surgery for refractory glaucoma.results and complications with a modified schocket technique. Arch Ophthalmol 1987; 105(4): 562-569.

3 Reichstein D, Kammer J, Recchia F. Combined 25-gauge vitrectomy and posterior tube shunt placement for advanced glaucoma. Ophthalmology 2011; 118(1): 77-81.

4 Hill RA, Heuer DK, Baerveldt G, Minckler DS, Martone JF. Molteno implantation for glaucoma in young patients. Ophthalmology 1991; 98(7): 1042-1046.

5 Lloyd MA, Sedlak T, Heuer DK, Minckler DS, Baerveldt G, Lee MB et al. Clinical experience with the single-plate molteno implant in complicated glaucomas. update of a pilot study. Ophthalmology 1992; 99(5): 679-687.

6 Tarantola RM, Agarwal A, Lu P, Joos KM. Long-term results of combined endoscope-assisted pars planavitrectomy and glaucoma tube shunt surgery. Retina 2011; 31(2): 275-283.

7 Vajaranant TS, Blair MP, McMahon T, Wilensky JT, de la Cruz J. Special considerations for pars plana tube-shunt placement in boston type 1 keratoprosthesis. Arch Ophthalmol 2010; 128(11): 1480-1482.

8 Tsai JH, Freeman JM, Chan CC, Schwartz GS, Derby EA, Petersen MR et al. A progressive anterior fibrosis syndrome in patients with postsurgical congenital aniridia. Am J Ophthalmol 2005; 140(6): 1075-1079.

9 Arroyave CP, Scott IU, Gedde SJ, Parrish 2nd RK, Feuer WJ. Use of glaucoma drainage devices in the management of glaucoma associated with aniridia. Am J Ophthalmol 2003; 135(2): 155-159.

10 Tsai JH, Derby E, Holland EJ, Khatana AK. Incidence and prevalence of glaucoma in severe ocular surface disease. Cornea 2006; 25(5): 530-532.

11 Varma R, Heuer DK, Lundy DC, Baerveldt G, Lee PP, Minckler DS. Pars planabaerveldt tube insertion with vitrectomy in glaucomas associated with pseudophakia and aphakia. Am J Ophthalmol 1995; 119(4): 401-407.

12 Scott IU, Alexandrakis G, Flynn Jr HW, Smiddy WE, Murray TG, Schiffman J et al. Combined pars planavitrectomy and glaucoma drainage implant placement for refractory glaucoma. Am J Ophthalmol 2000; 129(3): 334-341.

13 Jeong HS, Nam DH, Paik HJ, Lee DY. Pars plana ahmed implantation combined with 23-gauge vitrectomy for refractory neovascular glaucoma in diabetic retinopathy. Korean J Ophthalmol 2012; 26(2): 92-96.

14 Lloyd MA, Heuer DK, Baerveldt G, Minckler DS, Martone JF, Lean JS et al. Combined molteno implantation and pars planavitrectomy for neovascularglaucomas. Ophthalmology 1991; 98(9): 1401-1405.

15 Bakhtiari P, Chan C, Welder JD, de la Cruz J, Holland EJ, Djalilian AR. Surgical and visual outcomes of the type I bostonkeratoprosthesis for the management of aniridic fibrosis syndrome in congenital aniridia. Am J Ophthalmol 2012; 153(5): 967-971.e2. 
16 Panarelli JF, Ko A, Sidoti PA, Garcia JP, Banitt MR. Angle closure after Boston keratoprosthesis. J Glaucoma 2013; 22: 725-729.

17 Kaynak S, Tekin NF, Durak I, Berk AT, Saatci AO, Soylev MF. Pars planavitrectomy with pars plana tube implantation in eyes with intractable glaucoma. Br J Ophthalmol 1998; 82(12): 1377-1382.

18 Joos KM, Lavina AM, Tawansy KA, Agarwal A. Posterior repositioning of glaucoma implants for anterior segment complications. Ophthalmology 2001; 108(2): 279-284.

19 Sidoti PA, Mosny AY, Ritterband DC, Seedor JA. Pars plana tube insertion of glaucoma drainage implants and penetrating keratoplasty in patients with coexisting glaucoma and corneal disease. Ophthalmology 2001; 108(6): 1050-1058.

20 Sheppard JD, Shrum KR. Parsplanamolteno implantation in complicated inflammatory glaucoma. Ophthalmic Surg 1995; 26(3): 218-222.

21 Gedde SJ, Schiffman JC, Feuer WJ, Herndon LW, Brandt JD, Budenz DL et al. Three-year follow-up of the tube versus trabeculectomy study. Am J Ophthalmol 2009; 148(5): 670-684.

22 Melamed S, Cahane M, Gutman I, Blumenthal M. Postoperative complications after molteno implant surgery. Am J Ophthalmol 1991; 111(3): 319-322. 